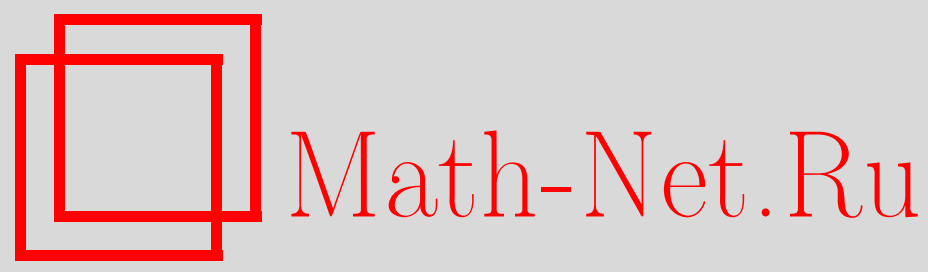

А. Ф. Измаилов, $\mathrm{K}$ условиям оптимальности в экстремальных задачах с нерегулярными ограниченияминеравенствами, Матем. заметки, 1999, том 66, выпуск 1, 89-101

DOI: https://doi.org/10.4213/mzm1144

Использование Общероссийского математического портала Math-Net.Ru подразумевает, что вы прочитали и согласны с пользовательским соглашением http://www . mathnet.ru/rus/agreement

Параметры загрузки:

IP: 54.210 .77 .194

26 апреля 2023 г., 14:38:32

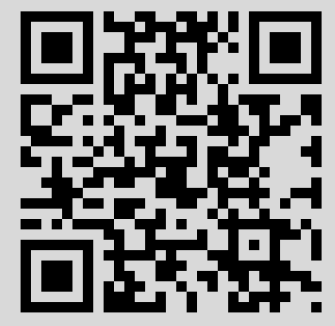




\section{К УСЛОВИЯМ ОПТИМАЛЬНОСТИ В ЭКСТРЕМАЛЬНЫХ ЗАДАЧАХ С НЕРЕГУЛЯРНЫМИ ОГРАНИЧЕНИЯМИ-НЕРАВЕНСТВАМИ}

\section{А. Ф. Измаилов}

Предлагается содержательная характеризация локальных решений экстремальных задач, ограничения которых, задаваемые с помощью вьпуклого конуса с непустой внутренностью, не предполагаются регулярньми. Основой проводимых построений служит описание касательно конуса к допустимому множеству задачи в исследуемой точке.

Библиография: 11 названий.

1. Введение. Вспомогательные результаты. Известно, что классические необходимые условия оптимальности в экстремальных задачах с ограничениями содержательны лишь при выполнении тех или иных условий регулярности (см., например, [1]-[3]). Специальные условия оптимальности для задач с нерегулярными ограничениями-равенствами, основанные на использовании так назьваемой конструкции 2-регулярности, предложены в работах [4]-[7] (см. также [2], [3]). Однако, попытки распространить эти построения (с помощью тех или иных известных приемов) на случай, когда в задаче имеются ограничения-неравенства, приводят к весьма грубым результатам, поскольку такие ограничения имеют определенную специфику. В этой связи выделим работу [7], где рассматривается случай, ког да ограничения-неравенства не вносят в задачу “дополнительной нерегулярности”, а также монографию [3], где построена содержательная теория условий второго порядка оптимальности, в том числе и для нерегулярных задач, среди ограничений которых имеются неравенства.

Настоящая статья является развитием работы [8], где предложен специальный подход к локальному исследованию экстремальных задач с нерегулярными ограничениями-неравенствами. Основные результаты из [8] содержатся в излагаемом ниже матераиале, причем в форме, сушественно более удобной для восприятия.

Пусть $X$ и $Y$ - линейные нормированные пространства. Речь будет идти о задаче

$$
\begin{gathered}
f(x) \rightarrow \min , \quad x \in D, \\
D=\{x \in X \mid F(x) \in K\},
\end{gathered}
$$

где функция $f: X \rightarrow \mathbb{R}$ и отображение $F: X \rightarrow Y$ обладают оговариваемой ниже гладкостью, а $K$ - вьпукльй конус в $Y$.

Работа выполнена при финансовой поддержке Российского фонда фундаментальных исследований (гранты № 96-01-00288 и 97-01-00188) 
Приведем некоторые вспомогательные факты, которые будут полезны при изучении локального строения множества $D$, заданного в (2) (доказательства опустим - все эти факты очевидно следуют из известных результатов выпуклого анализа [1], [9]-[11]).

Для конуса $C$ и произвольного множества $\Xi$ в линейном топологическом пространстве $\Sigma$ стандартным образом определим сопряженный конус

$$
C^{*}=\left\{\sigma^{*} \in \Sigma^{*} \mid\left\langle\sigma^{*}, \sigma\right\rangle \geqslant 0 \quad \forall \sigma \in C\right\}
$$

и аннулятор

$$
\Xi^{\perp}=\left\{\sigma^{*} \in \Sigma^{*} \mid\left\langle\sigma^{*}, \sigma\right\rangle=0 \quad \forall \sigma \in \Xi\right\}
$$

соответственно, где $\Sigma^{*}$ - сопряженное к $\Sigma$ пространство, $\langle\cdot, \cdot\rangle$ - спаривание элементов пространств $\Sigma^{*}$ и $\Sigma$. Кроме того, для конуса $\widetilde{C}$ в $\Sigma^{*}$ положим

$$
{ }^{*} \widetilde{C}=\left\{\sigma \in \Sigma \mid\left\langle\sigma^{*}, \sigma\right\rangle \geqslant 0 \quad \forall \sigma^{*} \in \widetilde{C}\right\} .
$$

Если $\Xi$ и $\Sigma$ - линейные топологические пространства, $\Lambda: \Xi \rightarrow \Sigma$ - непрерывньй линейньй оператор, то $\Lambda^{*}: \Sigma^{*} \rightarrow \Xi^{*}-$ сопряженньй к $\Lambda$ оператор.

Через int $\Xi$ и $\mathrm{cl} \Xi$ будем обозначать соответственно внутренность и замыкание множества $\Xi$ (в соответствующей топологии), а через lin $\Xi$ и cone $\Xi$ - соответственно линейную и коническую оболочки этого множества (в соответствующем линейном пространстве; коническая оболочка понимается как минимальньй выпукльй конус, содержащий $\Xi)$.

Лемма 1. Пусть $\Sigma$ - линейное топологическое пространство, $C$ - выпуклый конус в $\Sigma$, int $C \neq \varnothing, L$ - линейное подпространство в $\Sigma$.

Тогда условие

$$
\operatorname{int} C \cap L=\varnothing
$$

равносильно условию

$$
C^{*} \cap L^{\perp} \neq\{0\} .
$$

Лемма 1 может рассматриваться как частньй случай теоремы Дубовицкого-Милютина о пересечении выпуклых конусов [1].

ЛЕмма 2. Пусть $\Sigma$ - локально выпуклое линейное топологическое пространство, $C_{1}$ и $C_{2}$ - непустые выпуклые конусы в $\Sigma$.

Тогда

$$
{ }^{*}\left(C_{1}^{*} \cap C_{2}^{*}\right)=\operatorname{cl}\left(C_{1}+C_{2}\right) .
$$

ЛЕмма 3. Пусть $\Sigma$ - линейное топологическое пространство, $\Xi_{1}$ и $\Xi_{2}$-выпуклые мнохсества в $\Sigma$, int $\Xi_{1} \neq \varnothing$.

Тогда

$$
\operatorname{int}\left(\Xi_{1}+\Xi_{2}\right)=\operatorname{int} \Xi_{1}+\Xi_{2}
$$

ЗАмЕчАниЕ 1. Ниже будет использоваться не сама лемма 3 , а такое ее следствие: если в условиях этой леммы пространство $\Sigma$ локально выпукло, то

$$
\operatorname{int} \operatorname{cl}\left(\Xi_{1}+\Xi_{2}\right)=\text { int } \Xi_{1}+\Xi_{2}
$$

Конус $C$ в линейном пространстве $\Sigma$ будем назьвать конечно порожденным, если либо он пуст, либо найдутся такие число $s \in \mathbb{N}$ и элементы $\sigma^{i} \in \Sigma, i=1, \ldots, s$, что

$$
\operatorname{cl} C=\operatorname{cone}\left\{\sigma^{1}, \ldots, \sigma^{s}\right\} \cup\{0\} .
$$


Лемма 4. Пусть $\Sigma$ - линейное нормированное пространство, $C_{1}$ и $C_{2}$-конечно порожденные конусы в $\Sigma$.

Tогда

$$
\operatorname{cl}\left(C_{1}+C_{2}\right)=\operatorname{cl} C_{1}+\operatorname{cl} C_{2}
$$

ЗАмЕчАниЕ 2. В левой части (4) стоит замкнутое множество, в то время как множество в правой части (4) без требования конечной порожденности конусов $C_{1}$ и $C_{2}$ может не быть замкнутым (см. пример 1 ниже). Поэтому без последнего требования лемма 4 неверна.

В связи с понятием конечно порожденного конуса приведем также следующий вариант леммы о сопряженном конусе.

ЛЕмма 5. Пусть $\Xi и \Sigma-$ линейные нормированные пространства, $\operatorname{dim} \Sigma<\infty$, $\Lambda: \Xi \rightarrow \Sigma-$ непрерывный линейный оператор, $C$-непустой замкнутый конечно порожденный конус в $\Sigma$.

Тогда для конуса $\Gamma=\{\xi \in \Xi \mid \Lambda \xi \in C\}$ имеет место равенство

$$
\Gamma^{*}=\Lambda^{*} C^{*} \text {. }
$$

ЗАМЕЧАнИЕ 3 . В условиях леммы 5 конус $C$ по необходимости является однородным полиэдром. Если предполагать, что $C$ - однородньй полиэдр, то требование конечномерности $\Sigma$ в лемме 5 можно снять (это сразу следует из леммы о сопряженном конуce $[1])$.

Наконец, отметим следующий факт, вытекающий из известного результата об отрезке для выпуклых множеств [9].

ЛЕМма 6. Пусть $\Sigma$ - локально выпуклое линейное топологическое пространство, $C-$ выпукльй конус в $\Sigma$.

Тогда для любых әлементов $\sigma^{1} \in \operatorname{cl} C u \sigma^{2} \in \operatorname{int} C$

$$
\sigma^{1}+\sigma^{2} \in \operatorname{int} C \text {. }
$$

2. Касательный конус. Пусть $X$ и $Y$ - линейные нормированные пространства. Напомним, что вектор $h \in X$ называется касательным к множеству $D \subset X$ в точке $x_{*} \in D$, если найдется такое отображение $r: \mathbb{R}_{+} \rightarrow X$, что

$$
\begin{gathered}
x_{*}+t h+r(t) \in D \quad \forall t \in \mathbb{R}_{+}, \\
\|r(t)\|=o(t) .
\end{gathered}
$$

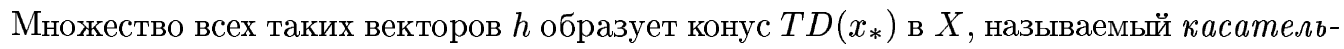
ным конусом к множеству $D$ в точке $x_{*}$. Конус $T D\left(x_{*}\right)$ всегда непуст (так как содержит 0) и замкнут [9].

Вопрос о возможно более точном описании касательного конуса к допустимому множеству экстремальной задачи в исследуемой точке является цетральным при построении условий оптимальности.

Далее считаем, что множество $D$ задано формулой (2), где $F: X \rightarrow Y$ - дифференцируемое по $\Phi$ реше в точке $x_{*} \in D$ отображение, а $K$ - вьпуклый конус в $Y$, причем

$$
\text { int } K \neq \varnothing \text {. }
$$


Для описания $T D\left(x_{*}\right)$ предлагается следуюшая конструкция.

Если $F\left(x_{*}\right) \in \operatorname{int} K$, то, очевидно, $T D\left(x_{*}\right)=X$. Пусть $F\left(x_{*}\right) \notin$ int $K$. Тогда int $K \cap$ $\operatorname{lin}\left\{F\left(x_{*}\right)\right\}=\varnothing$, что согласно лемме 1 равносильно условию

$$
\widetilde{C}_{0}\left(x_{*}\right) \neq\{0\},
$$

где

$$
\widetilde{C}_{0}\left(x_{*}\right)=K^{*} \cap\left\{F\left(x_{*}\right)\right\}^{\perp}
$$

- конус в $Y^{*}$. Согласно лемме 2

$$
{ }^{*} \widetilde{C}_{0}\left(x_{*}\right)=\operatorname{cl}\left(K+\operatorname{lin}\left\{F\left(x_{*}\right)\right\}\right),
$$

причем (8) равносильно условию

$$
{ }^{*} \widetilde{C}_{0}\left(x_{*}\right) \neq Y .
$$

Займемся необходимыми условиями касания. Для любого вектора $h \in T D\left(x_{*}\right)$ и любого $y^{*} \in K^{*}$ из $(2),(5),(6)$ имеем

$$
0 \leqslant\left\langle y^{*}, F\left(x_{*}+t h+r(t)\right)\right\rangle=\left\langle y^{*}, F\left(x_{*}\right)\right\rangle+t\left\langle y^{*}, F^{\prime}\left(x_{*}\right) h\right\rangle+o(t) \quad \forall t \in \mathbb{R}_{+},
$$

откуда согласно (9) немедленно вытекает необходимое условие первого порядка касания

$$
F^{\prime}\left(x_{*}\right) h \in{ }^{*} \widetilde{C}_{0}\left(x_{*}\right) .
$$

Отметим, что это условие содержательно в силу (11). Отметим также, что согласно $(7),(10)$ и замечанию 1

$$
\operatorname{int}{ }^{*} \widetilde{C}_{0}\left(x_{*}\right)=\operatorname{int} K+\operatorname{lin}\left\{F\left(x_{*}\right)\right\} \neq \varnothing .
$$

ЗАМЕЧАниЕ 4. В некоторых случаях полезна следующая наглядная интерпретация полученного необходимого условия касания:

$$
\begin{gathered}
T D\left(x_{*}\right) \subset\left\{h \in X \mid F^{\prime}\left(x_{*}\right) h \in T K\left(F\left(x_{*}\right)\right)\right\}, \\
T K\left(F\left(x_{*}\right)\right)=\operatorname{cl}\left(K+\operatorname{lin}\left\{F\left(x_{*}\right)\right\}\right) .
\end{gathered}
$$

Перейдем к достаточным условиям касания. Пусть теперь вектор $h \in X$ удовлетворяет $(12)$. Если $F^{\prime}\left(x_{*}\right) h \in \operatorname{int}{ }^{*} \widetilde{C}_{0}\left(x_{*}\right)$, то в силу (13) найдутся такие $y \in \operatorname{int} K$ и $\lambda \in \mathbb{R}$, что $F^{\prime}\left(x_{*}\right) h=y+\lambda F\left(x_{*}\right)$. Поэтому для любого достаточно малого числа $t>0$ согласно лемме 6 имеем

$$
F\left(x_{*}+t h\right)=(1+t \lambda) F\left(x_{*}\right)+t y+\omega_{1}(t) \in \operatorname{int} K
$$

(здесь $\left.\omega_{1}: \mathbb{R}_{+} \rightarrow Y,\left\|\omega_{1}(t)\right\|=o(t)\right)$, и, в частности, в силу $(2) h \in T D\left(x_{*}\right)$. Последнее останется верным и в случае, когда $F^{\prime}\left(x_{*}\right) h \notin$ int ${ }^{*} \widetilde{C}_{0}\left(x_{*}\right)$, но существует такая сходящаяся к $h$ последовательность $\left\{h^{k}\right\} \subset X$, что $\left\{F^{\prime}\left(x_{*}\right) h^{k}\right\} \subset$ int ${ }^{*} \widetilde{C}_{0}\left(x_{*}\right)$ (это следует из замкнутости $\left.T D\left(x_{*}\right)\right)$. Если найдется хотя-бы один элемент $\bar{h} \in X$, удовлетворяющий условию

$$
F^{\prime}\left(x_{*}\right) \bar{h} \in \operatorname{int}^{*} \widetilde{C}_{0}\left(x_{*}\right),
$$


то нужную последовательность $\left\{h^{k}\right\}$ можно указать явно, положив, например, $h^{k}=$ $(1-1 / k) h+\bar{h} / k, k \in \mathbb{N}$. Действительно, при этом для любого $k \in \mathbb{N}$ в силу $(12),(14)$ и леммы 6 имеем

$$
F^{\prime}\left(x_{*}\right) h^{k}=\left(1-\frac{1}{k}\right) F^{\prime}\left(x_{*}\right) h+\frac{1}{k} F^{\prime}\left(x_{*}\right) \bar{h} \in \operatorname{int}^{*} \widetilde{C}_{0}\left(x_{*}\right)
$$

Условие существания элемента $\bar{h} \in X$, удовлетворяющего (14), равносильно условию

$$
\operatorname{int}^{*} \widetilde{C}_{0}\left(x_{*}\right) \cap \operatorname{Im} F^{\prime}\left(x_{*}\right) \neq \varnothing
$$

которое естественно назвать условием регулярности (первого порядка) заданного в (2) множества $D$ в точке $x_{*} \in D$. Это - класическое условие регулярности типа условия Мангасаряна-Фрамовица [1]. Именно наличие такого условия делает содержательными классические необходимые условия оптимальности (принцип Лагранжа) для экстремальных задач рассматриваемого класса.

ЗАмЕчаниЕ 5 . Если отображение $F$ регулярно в точке $x_{*} \in D$ в том смысле, что $\operatorname{Im} F^{\prime}\left(x_{*}\right)=Y$ [1], [2], [9], то условие (15), очевидно, выполнено в силу (13). Поэтому введенное условие регулярности множества $D$ слабее условия регулярности отображения $F$.

В дальнейшем потребуется очевидное усиление полученного результата, связанное с тем, что условие линейности ограничений само является условием регулярности [11] никаких дополнительных условий регулярности для таких ограничений не требуется.

Теорема 1. Пусть $X, Y$ и $Z$ - линейные нормированные пространства, $K$ и $C$ выпуклые конусы в $Y$ и $Z$ соответственно, причем К удовлетворяет условию (7). Пусть множество $D$ задано формулой (2), әде $F: X \rightarrow Y$ - дифферениируемое по Фреше в точке $x_{*} \in D$ отображсение. Пусть $A: X \rightarrow Z$ - непрерывный линейный onepamop, $\Delta=\{x \in D \mid A x \in C\}$.

Тогда

1) всякий вектор $h \in T \Delta\left(x_{*}\right)$ удовлетворяет условию (12), причем

$$
A h \in \operatorname{cl}\left(C+\operatorname{lin}\left\{A x_{*}\right\}\right)
$$

2) если для вектора $h \in X$ выполнено (12) и условие

$$
A h \in C+\operatorname{lin}\left\{A x_{*}\right\}
$$

причем существует әлемент $\bar{h} \in X$, удовлетворяющий (14) и условию

$$
A \bar{h} \in C+\operatorname{lin}\left\{A x_{*}\right\}
$$

$m o h \in T \Delta\left(x_{*}\right)$. 
ЗАмЕчАнИЕ 6 . Если в условиях теоремы 1 конус $C$ - замкнутьй и конечно порожденньй, то с помощью лемм 2 и 4 легко получаем, что четыре условия (16), (17),

$$
A h \in T C\left(A x_{*}\right)
$$

и

$$
h \in T\{x \in X \mid A x \in C\}\left(x_{*}\right)
$$

на элемент $h \in X$ равносильны. Аналогичную интерпретацию в этом случае допускает и условие (18) на элемент $\bar{h} \in X$.

Продолжим изучение конуса $T D\left(x_{*}\right)$. В нерегулярном случае, когда $(15)$ не вьполнено, этот конус может быть у́же, чем множество векторов $h \in X$, удовлетворяющих (12), и для получения более точного описания представляется естественным привлекать информацию о старших производных отображения $F$. Этому и посвящена остальная часть пункта. Далее предполагаем, что отображение $F$ дважды дифференцируемо по Фреше в точке $x_{*}$.

Введем в $Y^{*}$ конус

$$
\widetilde{C}_{1}\left(x_{*}\right)=\left({ }^{*} \widetilde{C}_{0}\left(x_{*}\right)\right)^{*} \cap\left(\operatorname{Im} F^{\prime}\left(x_{*}\right)\right)^{\perp}
$$

Согласно (10)

$$
\left({ }^{*} \widetilde{C}_{0}\left(x_{*}\right)\right)^{*}=\left(\operatorname{cl}\left(K+\operatorname{lin}\left\{F\left(x_{*}\right)\right\}\right)\right)^{*}=\left(K+\operatorname{lin}\left\{F\left(x_{*}\right)\right\}\right)^{*}=K^{*} \cap\left\{F\left(x_{*}\right)\right\}^{\perp}
$$

поэтому

$$
\widetilde{C}_{1}\left(x_{*}\right)=K^{*} \cap\left\{F\left(x_{*}\right)\right\}^{\perp} \cap\left(\operatorname{Im} F^{\prime}\left(x_{*}\right)\right)^{\perp}
$$

и с учетом леммы 2

$$
{ }^{*} \widetilde{C}_{1}\left(x_{*}\right)=\operatorname{cl}\left(K+\operatorname{lin}\left\{F\left(x_{*}\right)\right\}+\operatorname{Im} F^{\prime}\left(x_{*}\right)\right) .
$$

В силу (19) и леммы 1 невьполнение (15) равносильно условию

$$
\widetilde{C}_{1}\left(x_{*}\right) \neq\{0\}
$$

(здесь учтено (13)). В свою очередь, (22) равносильно условию

$$
{ }^{*} \widetilde{C}_{1}\left(x_{*}\right) \neq Y .
$$

Для любого вектора $h \in T D\left(x_{*}\right)$ и любого $y^{*} \in K^{*}$ из $(2),(5),(6)$ имеем

$$
\begin{aligned}
0 \leqslant & \left\langle y^{*}, F\left(x_{*}+t h+r(t)\right)\right\rangle=\left\langle y^{*}, F\left(x_{*}\right)\right\rangle \\
& +\left\langle y^{*}, F^{\prime}\left(x_{*}\right)(t h+r(t))\right\rangle+\frac{t^{2}}{2}\left\langle y^{*}, F^{\prime \prime}\left(x_{*}\right)[h]^{2}\right\rangle+o\left(t^{2}\right) \quad \forall t \in \mathbb{R}_{+},
\end{aligned}
$$

откуда согласно (20) немедленно вытекает необходимое условие второго порядка касания

$$
F^{\prime \prime}\left(x_{*}\right)[h]^{2} \in{ }^{*} \widetilde{C}_{1}\left(x_{*}\right),
$$

содержательное в нерегулярном случае в силу (23). Отметим, что согласно (7), (21) и замечанию 1

$$
\text { int }{ }^{*} \widetilde{C}_{1}\left(x_{*}\right)=\operatorname{int} K+\operatorname{lin}\left\{F\left(x_{*}\right)\right\}+\operatorname{Im} F^{\prime}\left(x_{*}\right) \neq \varnothing .
$$


Перейдем к достаточным условиям касания. Пусть теперь вектор $h \in X$ удовлетворяет (12), (24). Более того, предположим, что

$$
F^{\prime}\left(x_{*}\right) h \in \operatorname{cl} K+\operatorname{lin}\left\{F\left(x_{*}\right)\right\}
$$

т.е. существуют такие $y^{1} \in \operatorname{cl} K$ и $\lambda_{1} \in \mathbb{R}$, что $F^{\prime}\left(x_{*}\right) h=y^{1}+\lambda_{1} F\left(x_{*}\right)$. Если $F^{\prime \prime}\left(x_{*}\right)[h]^{2} \in \operatorname{int}^{*} \widetilde{C}_{1}\left(x_{*}\right)$, то согласно (25) найдутся такие $y^{2} \in \operatorname{int} K, \lambda_{2} \in \mathbb{R}$ и $\bar{x} \in X$, что $F^{\prime \prime}\left(x_{*}\right)[h]^{2}=y^{2}+\lambda_{2} F\left(x_{*}\right)+F^{\prime}\left(x_{*}\right) \bar{x}$. Поэтому для любого достаточно малого числа $t>0$ согласно лемме 6 имеем

$$
\begin{aligned}
F\left(x_{*}+t h-\frac{t^{2}}{2} \bar{x}\right)= & F\left(x_{*}\right)+t\left(y^{1}+\lambda_{1} F\left(x_{*}\right)\right) \\
& -\frac{t^{2}}{2} F^{\prime}\left(x_{*}\right) \bar{x}+\frac{t^{2}}{2}\left(y^{2}+\lambda_{2} F\left(x_{*}\right)+F^{\prime}\left(x_{*}\right) \bar{x}\right)+\omega_{2}(t) \\
= & \left(1+t \lambda_{1}+\frac{t^{2}}{2} \lambda_{2}\right) F\left(x_{*}\right)+t y^{1}+\frac{t^{2}}{2} y^{2}+\omega_{2}(t) \in \operatorname{int} K
\end{aligned}
$$

$\left(\right.$ здесь $\left.\omega_{2}: \mathbb{R}_{+} \rightarrow Y,\left\|\omega_{2}(t)\right\|=o\left(t^{2}\right)\right)$ и, в частности, в силу $(2) h \in T D\left(x_{*}\right)$.

ЗАмЕчАниЕ 7 . Если конус $K$ - конечно порожденный, то из (10) и леммы 4 вытекает эквивалентность условий (12) и (26) на элемент $h \in X$. В общем случае заменить в проведенном рассуждении условие (26) более слабым условием (12) нельзя.

ПримеР 1. Пусть $X=\mathbb{R}, Y=\mathbb{R}^{3}$,

$$
\begin{gathered}
K=\text { cone }\left\{\left.y \in \mathbb{R}^{3}\left|y_{1}=1, y_{3}=\right| y_{2}\right|^{3 / 2}\right\}, \\
F: \mathbb{R} \rightarrow \mathbb{R}^{3}, \quad F(x)=\left(\begin{array}{c}
1 \\
x \\
x^{2}
\end{array}\right) .
\end{gathered}
$$

Для точки $x_{*}=0 \in \mathbb{R}$ имеем: $F(0) \in K,{ }^{*} \widetilde{C}_{0}(0)={ }^{*} \widetilde{C}_{1}(0)=\left\{y \in \mathbb{R}^{3} \mid y_{3} \geqslant 0\right\}$, и, как нетрудно убедиться, для элемента $h=1 \in \mathbb{R}$ вьполняются условия $F^{\prime}(0) h \in{ }^{*} \widetilde{C}_{0}(0)$, $F^{\prime \prime}(0)[h]^{2} \in \operatorname{int}^{*} \widetilde{C}_{1}(0)$. Вместе с тем, очевидно, 0 - изолированная точка множества $D$, задаваемого формулой $(2)$, а значит, $T D(0)=\{0\}$.

Если $F^{\prime \prime}\left(x_{*}\right)[h]^{2} \notin \operatorname{int}{ }^{*} \widetilde{C}_{1}\left(x_{*}\right)$, но существует такая сходящаяся к $h$ последовательность $\left\{h^{k}\right\} \subset X$, что $\left\{F^{\prime \prime}\left(x_{*}\right)\left[h^{k}\right]^{2}\right\} \subset$ int ${ }^{*} \widetilde{C}_{1}\left(x_{*}\right)$, то по-прежнему $h \in T D\left(x_{*}\right)$ в силу замкнутости $T D\left(x_{*}\right)$. В частности, если найдется хотя-бы один такой элемент $\bar{h} \in X$, что

$$
\begin{gathered}
F^{\prime}\left(x_{*}\right) \bar{h} \in \operatorname{cl} K+\operatorname{lin}\left\{F\left(x_{*}\right)\right\}, \\
F^{\prime \prime}\left(x_{*}\right)[h, \bar{h}] \in \operatorname{int}^{*} \widetilde{C}_{1}\left(x_{*}\right),
\end{gathered}
$$

то нужную последовательность $\left\{h^{k}\right\}$ можно указать явно, вновь положив $h^{k}=$ $(1-1 / k) h+\bar{h} / k, k \in \mathbb{N}$. Действительно, при этом для любого $k \in \mathbb{N}$ из (26), (27) выводим

$$
F^{\prime}\left(x_{*}\right) h^{k}=\left(1-\frac{1}{k}\right) F^{\prime}\left(x_{*}\right) h+\frac{1}{k} F^{\prime}\left(x_{*}\right) \bar{h} \in \operatorname{cl} K+\operatorname{lin}\left\{F\left(x_{*}\right)\right\} .
$$


Кроме того, для любого достаточно большого $k \in \mathbb{N}$ в силу $(24),(28)$ и леммы 6 имеем

$$
\begin{aligned}
F^{\prime \prime}\left(x_{*}\right)\left[h^{k}\right]^{2}= & \left(1-\frac{1}{k}\right)^{2} F^{\prime \prime}\left(x_{*}\right)[h]^{2} \\
& +\frac{1}{k}\left(2\left(1-\frac{1}{k}\right) F^{\prime \prime}\left(x_{*}\right)[h, \bar{h}]+\frac{1}{k} F^{\prime \prime}\left(x_{*}\right)[\bar{h}]^{2}\right) \in \operatorname{int}^{*} \widetilde{C}_{1}\left(x_{*}\right) .
\end{aligned}
$$

ОПРЕДЕЛЕНИЕ. Заданное в (2) множество $D$ будем назьвать 2-регулярным в точке $x_{*} \in D$ на элементе $h \in X$, если существует элемент $\bar{h} \in X$, удовлетворяющий $(27),(28)$.

ЗАмЕчаниЕ 8. Подчеркнем, что если конус $K$ - конечно порожденный, то согласно (10) и лемме 4 условие $(27)$ на элемент $\bar{h} \in X$ равносильно условию

$$
F^{\prime}\left(x_{*}\right) \bar{h} \in{ }^{*} \widetilde{C}_{0}\left(x_{*}\right) .
$$

Суммируя сказанное, сформулируем основной результат о касательном конусе.

ТЕОрема 2. Пусть $X$ и $Y$ - линейные нормированные пространства, $K$ - выпуклый конус в $Y$, удовлетворяющий условию (7). Пусть множество $D$ задано формулой (2), где $F: X \rightarrow Y-$ дважсды дифферениируемое по Фреше в точке $x_{*} \in D$ отобрахсение.

Тогда

1) всякий вектор $h \in T D\left(x_{*}\right)$ удовлетворяет условиям $(12),(24)$;

2) если множество $D$ 2-регулярно в точке $x_{*}$ на әлементе $h \in X$, который удовлетворяет условиям (24), (26), то $h \in T D\left(x_{*}\right)$.

В работе [8] содержится ряд примеров, которые могут использоваться для иллюстрации теоремы 1 в случае, когда $Y=\mathbb{R}^{m}, K=\mathbb{R}_{+}^{m}, m \in \mathbb{N}$ (см. также замечание 11 и пример 2 ниже).

ЗАмечание 9. Разумеется, теорема 1 описывает и регулярный случай. Если в ее условиях вьполнено (15), то в силу (19) и леммы $1 \widetilde{C}_{1}\left(x_{*}\right)=\{0\},{ }^{*} \widetilde{C}_{1}\left(x_{*}\right)=Y$, и условие (24) вьполняется тривиальным образом для любого элемента $h \in X$. Более того, если $h$ удовлетворяет (26), то при $\bar{h}=h$ вьполняются и условия $(27),(28)$ (в частности, это всегда будет так при $h=0)$.

ЗАмЕчАнИЕ 10 . Несложно убедиться, что если отображение $F$-регулярно в точке $x_{*} \in D$ на элементе $h \in X$ в смысле работ [2]-[7], то тем же свойством обладает и заданное в $(2)$ множество $D$. Поэтому введенное условие 2 -регулярности множества $D$ слабее условия 2-регулярности отображения $F$, которое, в свою очередь, слабее условия регулярности отображения $F$.

ЗАмЕчаниЕ 11 . Пусть $Y=\mathbb{R}^{m}, K=\mathbb{R}_{+}^{m}, m \in \mathbb{N}$, т.е. (1), (2)- стандартная задача с ограничениями-неравенствами. Тогда в силу (10) и лемм 3,4 для векторов $h, \bar{h} \in X$ условия (12) и (14) совпадают с условиями совместности систем линейньх неравенств

$$
\lambda F\left(x_{*}\right)+F^{\prime}\left(x_{*}\right) h \geqslant 0
$$

и

$$
\lambda F\left(x_{*}\right)+F^{\prime}\left(x_{*}\right) \bar{h}>0
$$


относительно $\lambda \in \mathbb{R}$ соответственно. Аналогично, в силу (10), (21) и лемм 3,4 условия $(24)$ и $(27),(28)$ совпадают с условиями совместности систем линейных неравенств

$$
\mu F\left(x_{*}\right)+F^{\prime}\left(x_{*}\right) x+F^{\prime \prime}\left(x_{*}\right)[h]^{2} \geqslant 0
$$

относительно $(\mu, x) \in \mathbb{R} \times X$ и

$$
\begin{array}{r}
\lambda F\left(x_{*}\right)+F^{\prime}\left(x_{*}\right) \bar{h} \geqslant 0, \\
\mu F\left(x_{*}\right)+F^{\prime}\left(x_{*}\right) x+F^{\prime \prime}\left(x_{*}\right)[h, \bar{h}]>0
\end{array}
$$

относительно $(\lambda, \mu, x) \in \mathbb{R} \times \mathbb{R} \times X$ соответственно.

3. Условия оптимальности. Теперь обратимся собственно к задаче (1), (2), где по-прежнему $X$ и $Y$ - линейные нормированные пространства, $K$-выпукльй конус в $Y$, удовлетворяющий условию (7), а $F: X \rightarrow Y$ - дважды дифференцируемое по Фреше в ислледуемой точке $x_{*} \in D$ отображение. Функция $f: X \rightarrow \mathbb{R}$ будет предполагаться дифференцируемой по $\Phi$ реше в точке $x_{*}$.

Используя обозначения п. 2 , введем в $X$ конусы

$$
\begin{aligned}
& H_{2}\left(x_{*}\right)=\left\{h \in X \mid F^{\prime}\left(x_{*}\right) h \in{ }^{*} \widetilde{C}_{0}\left(x_{*}\right), F^{\prime \prime}\left(x_{*}\right)[h]^{2} \in{ }^{*} \widetilde{C}_{1}\left(x_{*}\right)\right\}, \\
& \widetilde{H}_{2}\left(x_{*}\right)=\left\{h \in X \mid F^{\prime}\left(x_{*}\right) h \in \operatorname{cl} K+\operatorname{lin}\left\{F\left(x_{*}\right)\right\}, F^{\prime \prime}\left(x_{*}\right)[h]^{2} \in{ }^{*} \widetilde{C}_{1}\left(x_{*}\right)\right\}, \\
& \bar{H}_{2}\left(x_{*}\right)=\left\{h \in \widetilde{H}_{2}\left(x_{*}\right) \mid \exists \bar{h} \in X: F^{\prime}\left(x_{*}\right) \bar{h} \in \operatorname{cl} K+\operatorname{lin}\left\{F\left(x_{*}\right)\right\},\right. \\
&\left.F^{\prime \prime}\left(x_{*}\right)[h, \bar{h}] \in \operatorname{int}{ }^{*} \widetilde{C}_{1}\left(x_{*}\right)\right\} .
\end{aligned}
$$

Согласно сказанному выше

$$
\bar{H}_{2}\left(x_{*}\right) \cup\{0\} \subset \widetilde{H}_{2}\left(x_{*}\right) \subset H_{2}\left(x_{*}\right)
$$

Из теоремы 2 и известных общих условий первого порядка оптимальности в задачах условной оптимизации (см., например, теоремы 3 и 4 в [8]) вытекают следующие необходимые и достаточные условия оптимальности в задаче $(1),(2)$.

ТеОрема 3. Пусть в условиях теоремы 2 функиия $f: X \rightarrow \mathbb{R}$ дифференцируема по Фреше в точке $x_{*}$, которая является локальным минимумом задачи (1), (2).

Тогда

$$
\left\langle f^{\prime}\left(x_{*}\right), h\right\rangle \geqslant 0 \quad \forall h \in \bar{H}_{2}\left(x_{*}\right) .
$$

Теорема 4. Пусть в условиях теоремы $2 \operatorname{dim} X<\infty$, функция $f: X \rightarrow \mathbb{R} \partial и ф-$ ферениируема по Фреше в точке $x_{*} u$

$$
\left\langle f^{\prime}\left(x_{*}\right), h\right\rangle>0 \quad \forall h \in H_{2}\left(x_{*}\right) \backslash\{0\} .
$$

Тогда $x_{*}-$ изолированный локальный минимум задачи (1), (2). 
ЗАмЕчАниЕ 12 . В отличие от случая ограничений-равенств конус $H_{2}\left(x_{*}\right)$ может не содержать линейных подпространств, поэтому условие (31) непротиворечиво.

Условие (30) можно переписать в виде

$$
f^{\prime}\left(x_{*}\right) \in\left(\bar{H}_{2}\left(x_{*}\right)\right)^{*},
$$

что соответствует лагранжевой форме условий оптимальности. Однако, выгисление сопряженного конуса, стоящего в правой части (32), - крайне трудная задача. Даже в случае, когда заданное в (2) множество $D 2$-регулярно в точке $x_{*} \in D$ в том смысле, что левое включение в (29) вьполнено как равенство, эта задача вряд-ли может быть решена в общем виде, поскольку конус $\widetilde{H}_{2}\left(x_{*}\right)$ задается нелинейньми ограничениями и, вообще говоря, даже не является вьпуклым. Иными словами, “лемма об аннуляторе" (о сопряженном конусе), которую можно было-бы применить к конусу $\widetilde{H}_{2}\left(x_{*}\right)$, неизвестна.

Вместе с тем, в свете теорем 3 и 4 ясно, что в дальнейшем исследовании нуждаются свойства дифференциала целевой функции задачи главным образом на тех элементах $h \in \bar{H}_{2}\left(x_{*}\right)$, для которых неравенство в (30) выполняется как равенство. Оказьвается, для таких $h$ можно получить специальную локальную лагранжеву форму условий оптимальности, аналогичную той, которая для экстремальных задач с ограничениями-равенствами изложена в [2]-[7].

А именно, пусть в условиях теоремы 3 для элемента $h \in \bar{H}_{2}\left(x_{*}\right)$ выполнено равенство

$$
\left\langle f^{\prime}\left(x_{*}\right), h\right\rangle=0 .
$$

Далее будем предполагать, что конус $K$ - конечно порожденный. При этом согласно замечанию 7 правое включение в (29) вьполнено как равенство. Отсюда следует устойчивость свойства 2-регулярности множества $D$ в точке $x_{*}$ на элементе $h$ в том смысле, что найдется окрестность $U$ точки $h$ в $X$, для которой

$$
H_{2}\left(x_{*}\right) \cap U \subset \bar{H}_{2}\left(x_{*}\right) .
$$

Поэтому согласно (30)

$$
\left\langle f^{\prime}\left(x_{*}\right), \xi\right\rangle \geqslant 0 \quad \forall \xi \in H_{2}\left(x_{*}\right) \cap U .
$$

Но из (33), (34) очевидно следует, что

$$
\left\langle f^{\prime}\left(x_{*}\right), \xi\right\rangle \geqslant 0 \quad \forall \xi \in T\left(H_{2}\left(x_{*}\right)\right)(h),
$$

т.e.

$$
f^{\prime}\left(x_{*}\right) \in\left(T\left(H_{2}\left(x_{*}\right)\right)(h)\right)^{*} .
$$

Остается вычислить конус $T\left(H_{2}\left(x_{*}\right)\right)(h)$, а также сопряженньй к нему. Оказывается, эта задача может быть решены с помошь теоремы 1 и леммы 5 (дело в том, что вьполнение условия 2 -регулярности множества $D$ в точке $x_{*}$ на элементе $h$ гарантирует, что конус $T\left(H_{2}\left(x_{*}\right)\right)(h)$ задается линейными ограничениями).

Действительно, согласно $(10)$ конус ${ }^{*} \widetilde{C}_{0}\left(x_{*}\right)$ - замкнутьй и конечно порожденный. Поэтому из условия существования элемента $\bar{h} \in X$, удовлетворяющего (27), (28), согласно теореме 1 и замечанию 6 вытекает равенство

$$
\begin{gathered}
T\left(H_{2}\left(x_{*}\right)\right)(h)=\left\{\xi \in X \mid F^{\prime}\left(x_{*}\right) \xi \in \operatorname{cl}\left({ }^{*} \widetilde{C}_{0}\left(x_{*}\right)+\operatorname{lin}\left\{F^{\prime}\left(x_{*}\right) h\right\}\right),\right. \\
\left.F^{\prime \prime}\left(x_{*}\right)[h, \xi] \in \operatorname{cl}\left({ }^{*} \widetilde{C}_{1}\left(x_{*}\right)+\operatorname{lin}\left\{F^{\prime \prime}\left(x_{*}\right)[h]^{2}\right\}\right)\right\}
\end{gathered}
$$


Конус $\operatorname{cl}\left({ }^{*} \widetilde{C}_{0}\left(x_{*}\right)+\operatorname{lin}\left\{F^{\prime}\left(x_{*}\right) h\right\}\right)-$ замкнутый и конечно порожденный. Одновременное вьполнение условия конечной порожденности конуса $K$ и условия (7) предполагает, что $\operatorname{dim} Y<\infty$. В частности, $\operatorname{rang} F^{\prime}\left(x_{*}\right)<\infty$, поэтому из $(21)$ следует, что конус $\operatorname{cl}\left({ }^{*} \widetilde{C}_{1}\left(x_{*}\right)+\operatorname{lin}\left\{F^{\prime \prime}\left(x_{*}\right)[h]^{2}\right\}\right)$ - тоже замкнутый и конечно порожденньй. Но тогда из (36) и леммы 5 получаем равенство

$$
\begin{aligned}
\left(T\left(H_{2}\left(x_{*}\right)\right)(h)\right)^{*}= & \left(F^{\prime}\left(x_{*}\right)\right)^{*}\left(\left({ }^{*} \widetilde{C}_{0}\left(x_{*}\right)\right)^{*} \cap\left\{F^{\prime}\left(x_{*}\right) h\right\}^{\perp}\right) \\
& +\left(F^{\prime \prime}\left(x_{*}\right)[h]\right)^{*}\left(\left({ }^{*} \widetilde{C}_{1}\left(x_{*}\right)\right)^{*} \cap\left\{F^{\prime \prime}\left(x_{*}\right)[h]^{2}\right\}^{\perp}\right) .
\end{aligned}
$$

Из соотношений (10), (21), (35), (37) вытекает

ТЕОрема 5. Пусть в условиях теоремы $2 K$ - конечно порохсденый конус, функиия $f: X \rightarrow \mathbb{R}$ дифферениируема по Фреше в точке $x_{*}$, которая является локальным минимумом задачи (1), (2). Пусть для некоторого әлемента $h \in \bar{H}_{2}\left(x_{*}\right)$ выполнено (33).

Тогда найдутся такие функиионалы

$$
\begin{aligned}
& y_{1}^{*}=y_{1}^{*}(h) \in K^{*} \cap\left\{F\left(x_{*}\right)\right\}^{\perp} \cap\left\{F^{\prime}\left(x_{*}\right) h\right\}^{\perp}, \\
& y_{2}^{*}=y_{2}^{*}(h) \in K^{*} \cap\left\{F\left(x_{*}\right)\right\}^{\perp} \cap\left(\operatorname{Im} F^{\prime}\left(x_{*}\right)\right)^{\perp} \cap\left\{F^{\prime \prime}\left(x_{*}\right)[h]^{2}\right\}^{\perp},
\end{aligned}
$$

что

$$
f^{\prime}\left(x_{*}\right)=\left(F^{\prime}\left(x_{*}\right)\right)^{*} y_{1}^{*}+\left(F^{\prime \prime}\left(x_{*}\right)[h]\right)^{*} y_{2}^{*} .
$$

ЗАмечаниЕ 13. Еще раз подчеркнем, что в теореме 5 речь идет и задачах "с конечномерным образом" - пространство $Y$ по необходимости конечномерно.

ЗАмЕЧАниЕ 14. Теорема 5 является следствием теоремы 3 - никакие дополнительные экстремальные свойства, относящиеся исходной задаче $(1),(2)$, при обосновании теоремы 5 не привлекались.

ЗАмЕЧАнИЕ 15 . Если в условиях теоремы $5 F^{\prime \prime}\left(x_{*}\right)[h]^{2} \in$ int ${ }^{*} \widetilde{C}_{1}\left(x_{*}\right)$, то согласно лемме 1 и (21)

$$
K^{*} \cap\left\{F\left(x_{*}\right)\right\}^{\perp} \cap\left(\operatorname{Im} F^{\prime}\left(x_{*}\right)\right)^{\perp} \cap\left\{F^{\prime \prime}\left(x_{*}\right)[h]^{2}\right\}^{\perp}=\{0\} .
$$

Поэтому в силу (38), (39) представление (40) принимает вид

$$
\begin{gathered}
f^{\prime}\left(x_{*}\right)=\left(F^{\prime}\left(x_{*}\right)\right)^{*} y_{1}^{*}, \\
y_{1}^{*}=y_{1}^{*}(h) \in K^{*} \cap\left\{F\left(x_{*}\right)\right\}^{\perp} \cap\left\{F^{\prime}\left(x_{*}\right) h\right\}^{\perp} .
\end{gathered}
$$

В частности, согласно замечанию 9 это всегда будет так, если множество $D$ регулярно в точке $x_{*}$. В последнем случае согласно тому же замечанию 9 под действие теоремы 5 всегда попадает элемент $h=0$, для которого (42) принимает вид

$$
y_{1}^{*} \in K^{*} \cap\left\{F\left(x_{*}\right)\right\}^{\perp} .
$$

Соотношения (41), (43) дают содержательную форму классического принципа Лагранжа для задачи $(1),(2)$. В правой части (43) стоит нормальный конус к конусу $K$ в точке 
$F\left(x_{*}\right) \in K$. В терминологии стандартных задач с ограничениями-неравенствами включение $y_{1}^{*} \in K^{*}$ суть условие "неотрищательности" множителей Лагранжа, а включение $y_{1}^{*} \in\left\{F\left(x_{*}\right)\right\}^{\perp}$ - условие дополняющей нежесткости [1], [9], [11]. Таким образом, теорема 5 содержит в себе и классические необходимые условия первого порядка оптимальности. Отметим, что если $h \notin \operatorname{Ker} F^{\prime}\left(x_{*}\right)$, то согласно $(42)$ на множитель накладывается дополнительное нетривиальное условие $y_{1}^{*}(h) \in\left\{F^{\prime}\left(x_{*}\right) h\right\}^{\perp}$, и представление (41) для $f^{\prime}\left(x_{*}\right)$ в этом случае становится более точным.

ПримеР 2. Пусть $X=Y=\mathbb{R}^{2}, K=\mathbb{R}_{+}^{2}$. Рассмотрим семейство линейных функций

$$
f: \mathbb{R}^{2} \rightarrow \mathbb{R}, \quad f(x)=a x_{1}+b x_{2},
$$

где $a, b \in \mathbb{R}$ - параметры, и отображение

$$
F: \mathbb{R}^{2} \rightarrow \mathbb{R}^{2}, \quad F(x)=\left(\begin{array}{c}
x_{1} \\
\frac{1}{2}\left(x_{1}^{2}-x_{2}^{2}\right)
\end{array}\right) .
$$

Тогда для точки $x_{*}=0 \in \mathbb{R}^{2}$ имеем: $F(0)=0$, т.е. $0 \in D$, где множество $D$ задано в (2), причем это множество не регулярно в точке 0 . Принцип Лагранжа [1], [9], [11] для задачи (1), (2) в этой точке выполняется при любых значениях параметров $a$ и $b$ тривиальньп образом (с нулевым множителем, отвечающим минимизируемой функции). C помощью замечания 11 нетрудно убедиться, что здесь

$$
D=T D(0)=\bar{H}_{2}(0)=H_{2}(0)
$$

(что иллюстрирует теорему 2). Рассмотрим некоторые характерные значения параметров.

Если $a=1, b=-1$, то 0 - (неизолированньй) локальньй минимум задачи (1), (2). Очевидно,

$$
\left\langle f^{\prime}(0), h\right\rangle \geqslant 0 \quad \forall h \in D
$$

(что иллюстрирует теорему 3), причем, например, для элемента $h=(1,1) \in D$ в $(44)$ реализуется равенство. При удовлетворяющих (38), (39) множителях

$$
y_{1}^{*}=\left(\begin{array}{c}
0 \\
\alpha
\end{array}\right) \mathbb{R}^{2}, \quad \alpha \in \mathbb{R}_{+}, \quad y_{2}^{*}=\left(\begin{array}{l}
0 \\
1
\end{array}\right) \in \mathbb{R}^{2}
$$

для указанного $h$ имеет место представление (40) (что иллюстрирует теорему 5). Чтобы множитель $y_{1}^{*}$ определялся однозначно, нужно наложить на него естественное дополнительное требование $y_{1}^{*} \in\left(\operatorname{Ker}\left(F^{\prime}(0)\right)^{*}\right)^{\perp}=\operatorname{Im} F^{\prime}(0)$. Подчеркнем, что для $h \in$ $D \backslash \operatorname{lin}\{(1,1)\}$ соответствующее представление не имеет места. Это объясняется тем, что для таких элементов $h$ в (44) реализуется строгое неравенство.

Если $a=1, b=0$, то в (44) реализуется строгое неравенство для всякого $h \in D \backslash\{0\}$, и 0 - изолированный локальный минимум задачи $(1),(2)$ (что иллюстрирует теорему 4).

Наконец, если $a=0, b=1$, то условие (44) очевидно нарушается для тех элементов $h \in D$, у которых $h_{2}<0$. Для элемента $h=(1,0) \in D$ в (44) реализуется равенство, но представление (40) не имеет места ни при каких множителях $y_{1}^{*}, y_{2}^{*} \in \mathbb{R}^{2}$. Таким образом, как из теоремы 3 , так и из теоремы 5 следует, что точка 0 в этом случае не является локальньм минимумом задачи (1), (2).

В заключение отметим, что в отличие от регулярного случая распространение проведенных построений на задачи с ограничениями-равенствами и неравенствами, связанное с отказом от условия (7), весьма затруднительно (если вообще возможно). 


\section{СПИСОК ЦИТИРОВАННОЙ ЛИТЕРАТУРЫ}

[1] Алексеев В. М., Тихомиров В. М., Фомин С. В. Оптимальное управление. М.: Наука, 1979.

[2] Измаилов А. Ф., Третьяков А. А. Факторанализ нелинейных отображений. М.: Наука, 1994.

[3] Арутюнов А. В. Условия экстремума. Анормальные и вырожденные задачи. М.: Факториал, 1997.

[4] Третьяков А. А. Необходимые и достаточные условия оптимальности $P$-го порядка // ЖВМ и МФ. 1984. Т. 24. № 2. С. 203-209.

[5] Аваков Е.Р. Условия экстремума для гладких задач с ограничениями типа равенств // ЖВМ и МФ. 1985. Т. 25. № 5. С. 680-693.

[6] Белаш К.Н., Третьяков А. А. Методы решения вырожденных задач // ЖВМ и МФ. 1988. Т. 28. № 7. C. 1097-1102.

[7] Аваков Е.Р. Необходимые условия экстремума для гладких анормальных задач с ограничениями типа равенств и неравенств // Матем. заметки. 1989. Т. 45. №6. С. 3-11.

[8] Измаилов А. Ф. Условия оптимальности для вырожденньг экстремальных задач с ограничениями типа неравенств // ЖКВМ и МФ. 1994. Т. 34. №6. С. 837-854.

[9] Иоффе А. Д., Тихомиров В. М. Теория экстремальных задач. М.: Наука, 1974.

[10] Пшеничный Б. Н. Необходимые условия экстремума. М.: Наука, 1982.

[11] Сухарев А. Г., Тимохов А. В., Федоров В. В. Курс методов оптимизации. М.: Наука, 1986. 